\title{
Nonlinear Interference Analysis of Probabilistic Shaping vs. 4D Geometrically Shaped Formats
}

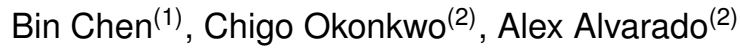

\begin{abstract}
(1) School of Computer Science and Information Engineering, Hefei University of Technology, China bin.chen@hfut.edu.cn

${ }^{(2)}$ Department of Electrical Engineering, Eindhoven University of Technology, The Netherlands
\end{abstract}

\begin{abstract}
Performance trade-offs between linear shaping and nonlinear tolerance of the recently introduced 4D orthant-symmetric 128-ary modulation format are investigated. Numerical simulations show 9.3\% reach increase with respect to the 7b4D-2A8PSK format and probabilistically-shaped 16QAM with short blocklength.
\end{abstract}

\section{Introduction}

In optical transmission systems, the performance of a given modulation format is determined by its tolerance to both linear amplified spontaneous emission (ASE) noise, and non-linear interference (NLI) arising from the Kerr effect. Designing modulation formats which increase achievable information rates (AIRs) in the presence of linear and nonlinear impairments is crucial to achieve higher capacity and longer reach.

NLI depends on the average power of the transmitted signal. One of the most widely used model for $\mathrm{NLI}$ is the so-called Gaussian noise (GN) model. The GN model neglects most of the specific properties of the transmitted signal, including its underlying modulation format [1]. However, in the vast majority of recent demonstrations, it has been shown that NLI also depends on the modulation format [2, 3]. A model considering modulation format dependency was later developed as an improvement to the GN model, known as enhanced GN (EGN) model [4, [5].

Signal shaping has recently been widely investigated in optical fibre communications to improve spectral efficiency (SE), and is currently implemented in commercial products via probabilistic shaping (PS) 6 and geometric shaping (GS) 7 . The performance of PS has been examined in theory, simulations, and experiments 8$]-12$. However, PS suffers from rate loss in practical implementations with finite blocklengths ${ }^{13}$ and also experiences increased NLI9],14]. Multidimensional (MD) geometric shaping based on constant modulus formats is known to offer NLI tolerance in the nonlinear channel[15, [16]. In so doing, the multidimensional modulation format "shapes out" the partial NLI at the expense of losing only partial degrees of freedom 17 .

In this paper, the recently proposed four- dimensional orthant-symmetric 128-ary (4DOS128) format 18 are studied that was designed by maximizing generalized mutual information with the benefit of significantly reducing the optimization searching space. We observe a performance trade-offs between linear shaping and nonlinear tolerance, and thus 4D-OS128 outperforms the corresponding nonlinearity-tolerant geometrically-shaped constellation 7b-4D2A8PSK and PS-16QAM with finite blocklength at the same SE.

\section{Orthant-Symmetric MD Geometric Shaping}

To solve the multi-parameter optimization challenges of MD geometric shaping and also to reduce the transceiver requirements, we proposed to impose an "orthant symmetry" constraint to the $N$-dimensional modulation format to be designed 18 . Orthant-symmetric labeled constellations are be generated from any first-orthant constellation, where the constellation points are obtained by folding the first-orthant points to the remaining orthants 18 .

In this paper, we focus on comparing modulation formats with a spectral efficiency (SE) of $m=$ 7 bit/4D-sym, which consists of $M=2^{m} N=4$ dimensional points $s_{i}, i \in\{1,2, \ldots, 128\}$ labeled by 7 bits $\boldsymbol{b}_{i}=\left[b_{1}, b_{2}, \ldots, b_{7}\right]$. For the $4 \mathrm{D}-\mathrm{OS} 128$ format, each orthant contains $2^{m-N}=8$ constellation points. The 8 constellation points in the first orthant are denoted by $\boldsymbol{t}_{j}=\left[t_{j 1}, t_{j 2}, t_{j 3}, t_{j 4}\right] \in \mathbb{R}_{+}^{4}$ with $j=\{1,2, \ldots, 8\}$. The first orthant is la-
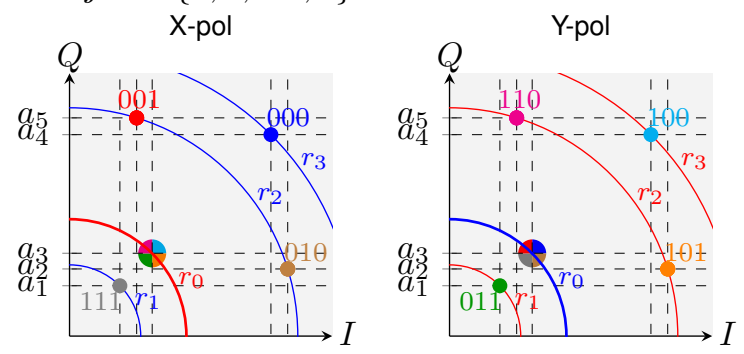

Fig. 1: First orthant of 4D-OS128 modulation formats and associated bit mapping $\left[b_{j 5}, b_{j 6}, b_{j 7}\right], j \in\{1,2, \ldots, 8\}$. 


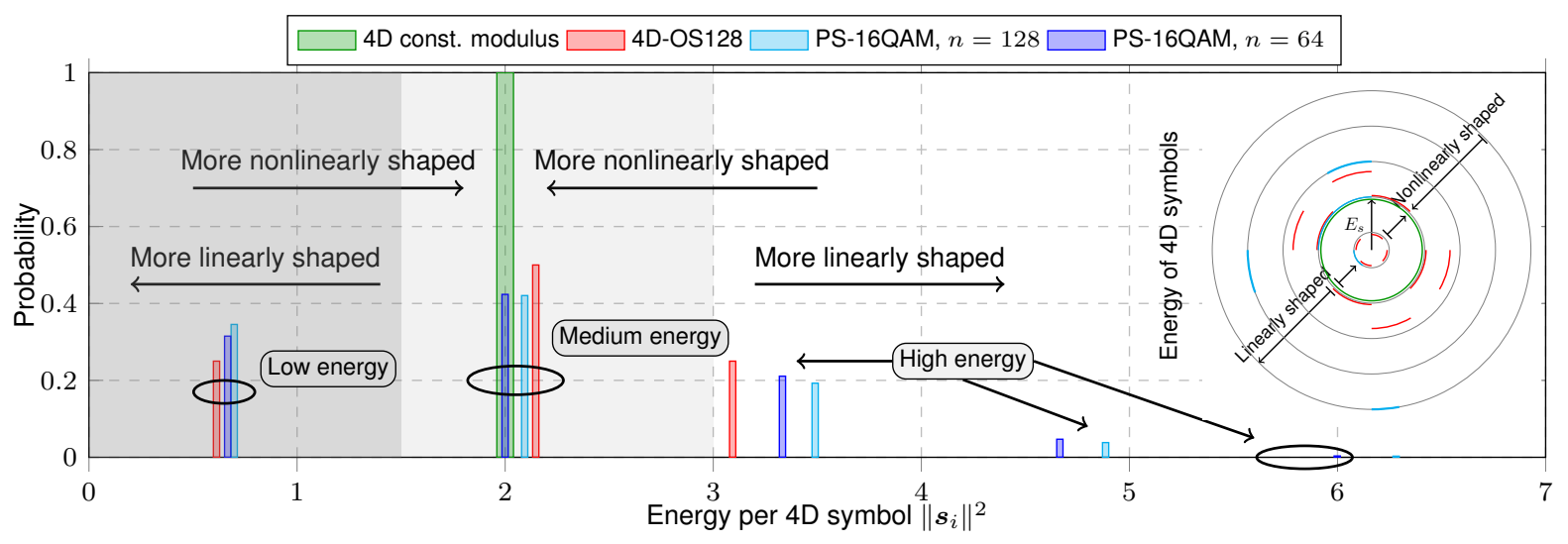

Fig. 2: Probability of energy per 4D symbol for four $\mathrm{SE}=7$ bits/4D-sym modulations. The variation of the transmitted symbols' energy contributes to the nonlinear interference noise (NLIN). Inset: 2D geometric representation of energy per 4D symbol.

beled by four binary bits $\left[b_{j 1}, b_{j 2}, b_{j 3}, b_{j 4}\right]$, which determine one of sixteen orthants. The remaining three bits $\left[b_{j 5}, b_{j 6}, b_{j 7}\right]$ determine one of eight point $t_{j}$ in the corresponding orthant. The 2D projections of the first orthant of the $4 \mathrm{D}-\mathrm{OS} 128^{[18]}$ format is shown in Fig. 1, where the symbols with the same color belong to the same 4D symbol. Due to the structure of the 4D-OS128 format, the 4D symbols have three energy levels $r_{0}^{2}+r_{k}^{2}, k \in$ $\{1,2,3\}$ (highlighted as three red or blue circle combinations in Fig. 11. The five amplitude values of the the 4D-OS128 format in Fig. 1 optimized for SNR of $9.5 \mathrm{~dB}$ are $\left(a_{1}, a_{2}, a_{3}, a_{4}, a_{5}\right)=$ $(0.2875,0.3834,0.4730,1.1501,1.2460)$.

\section{Probabilistic Amplitude Shaping}

In addition to the orthant-symmetric MD modulation format, we also consider probabilistic amplitude shaping (PAS) with quadrature amplitude modulation (QAM) to achieve the same SE of $7 \mathrm{bit} / 4 \mathrm{D}$-sym. In this paper, PS-16QAM is generated via probabilistically shaping polarizationmultiplexed (PM)-16QAM with constant composition distribution matching (CCDM). To take finitelength CCDM rate loss into account, the AIRs of PAS for bit-metric decoding (BMD) and for a finitelength CCDM of length $n$ is computed as ${ }^{[19]}$,

$$
\operatorname{AIR}_{n}=\left[H(\boldsymbol{C})-\sum_{i}^{m} H\left(C_{i} \mid Y\right)\right]-R_{\text {loss }, n},
$$

where $\boldsymbol{C}=\left(C_{1}, \ldots, C_{m}\right)$ represents the $m$ coded bit levels of the considered modulation format, $H(\cdot)$ denotes entropy, $R_{\text {loss }, n}$ indicate the finitelength rate loss $[20]$ and $Y$ is the channel output.

\section{Modulation-dependent NLI}

$\mathrm{NLI}$ in the EGN model is effectivel considered as additive white Gaussian noise. It is well understood that low energy variations in the signal reduce NLI21. Therefore, choosing symbols with less energy variations and close to the envelope of a four-dimensional ball can lead to less
Tab. 1: Simulation parameters.

\begin{tabular}{lr}
\hline Parameter name & Value \\
\hline Symbol rate & $41.79 \mathrm{GBd}$ \\
Root-raised-cosine roll-off factor & $1 \%$ \\
Channel frequency spacing & $50 \mathrm{GHz}$ \\
Center wavelength & $1550 \mathrm{~nm}$ \\
Attenuation & $0.21 \mathrm{~dB} / \mathrm{km}$ \\
Dispersion parameter & $16.9 \mathrm{ps} \mathrm{nm}^{-1} \mathrm{~km}^{-1}$ \\
Nonlinearity parameter & $1.31 \mathrm{~W}^{-1} \mathrm{~km}^{-1}$ \\
\hline
\end{tabular}

$\mathrm{NLI}$, which can be considered as nonlinear shaping. This is in contradiction with Gaussian shaped constellations choosing symbols within the multidimensional balls, which is referred as linear shaping.

Fig. 2 shows the example of linear shaping and nonlinear shaping by comparing the probability distribution of $4 \mathrm{D}$ symbol's energy for three different formats (all normalized to unit energy per polarization): 4D constant-modulus (CM) constellations, 4D-OS128 and PS-16QAM with block length of $n=64$ and $n=128$. We can observe that nonlinear shaping is in contradiction with linear shaping, which moves the constellation symbols away from the average energy $E_{s}$ (also see 2D geometric representation of energy per 4D symbol as inset in Fig. 2). In addition, the $4 \mathrm{D}$ constellation symbols' energy spread out from the average normalized energy $E_{s}=2$, which are divided as three groups: low energy symbols, medium energy symbols and high energy symbols. We will show in following section that this 4D energy distribution can induce NLI fluctuation for the nonlinear fibre channel.

\section{Numerical Simulations}

Split-step Fourier method simulations with a step size of $0.1 \mathrm{~km}$ were performed to compare the modulation formats and predict system performance. The simulation parameters are given in Table 1 for the optical multi-span fibre link under consideration, which comprises multiple standard single-mode fibre spans of $75 \mathrm{~km}$, amplified at the 


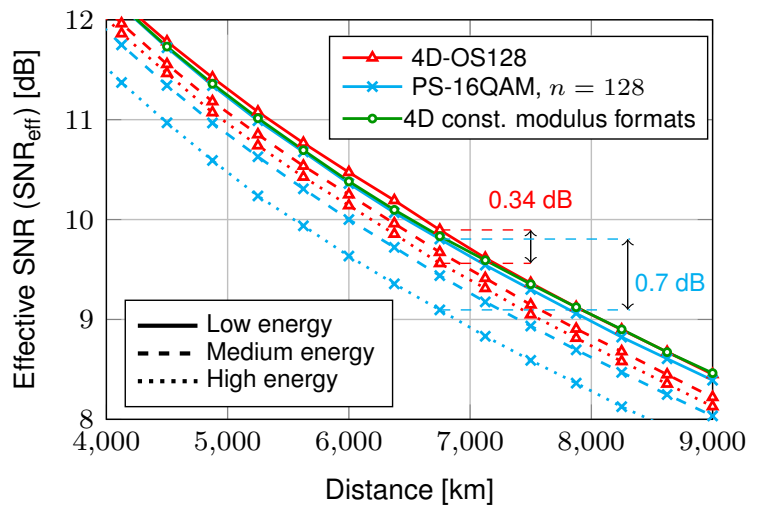

Fig. 3: $\mathrm{SNR}_{\mathrm{eff}}$ vs. transmission distance at $P_{\mathrm{ch}}=-1 \mathrm{dBm}$. end of each span by an EDFA with noise figure of $5 \mathrm{~dB}$. The encoded bits are mapped according to four modulation formats: PS-16QAM, constantmodulus 7b4D-2A8PSK, and 4D-OS128. Each of the 11 WDM channels carries independent data, where all of them are assumed to have the same transmitted power. An ideal receiver is used for detection and chromatic dispersion is digitally compensated.

In Fig. 3, the effective SNR (SNR eff $)$ in dB versus transmission distance for three modulation formats is evaluated. The $\mathrm{SNR}_{\text {eff }}$ is estimated from the transmitted data $X$ and received symbols $Y$ as $E_{s} / \sigma^{2}$ where $\sigma^{2}=\operatorname{var}(Y-X)$ denotes the noise variance. Fig. 3 shows the average SNRs calculated for the three energy levels: low, medium, and high (see Fig. 2). We observe that the $S_{N R} R_{\text {eff }}$ varies significantly with $4 \mathrm{D}$ symbols' energy for 4D-OS128 and PS-16QAM. At a distance of $6750 \mathrm{~km}$, the SNR difference between low and high energy symbols of PS-16QAM is $0.7 \mathrm{~dB}$, while the SNR difference for 4D-OS128 is only $0.34 \mathrm{~dB}$. The reason for this effect is that the transmitted symbols of 4D-OS128 are more close to a constant-modulus sequences than PS16QAM's. This observation permits us to assert that the proposed $4 \mathrm{D}-\mathrm{OS} 128$ is more robust than PS solutions to fiber nonlinear impairments, and thus, gives an intuition about optimal design and implementation of future MD formats.
In Fig. 4, we show the SNR and AIR of 7b4D2A8PSK, 4D-OS128 and PS-16QAM with DM blocklengths $n=32,64,128$. We observe that PS with $n=128$ gives slightly higher AIR, but the resulting increased rate loss diminishes the efficiency of DM as the blocklength decreases. With $n=32$ and $n=64$, PS-16QAM has even worse performance than 4D-OS128. These losses of PS-16QAM are shown in Fig. 4 (a) and (b), and are particularly visible in the highly nonlinear regime. For the considered optical fiber transmission setup, 4D-OS128 can achieve approximately $9.3 \%(700 \mathrm{~km})$ of reach increase with respect to 7b4D-2A8PSK and PS-16QAM with blocklength $n=32$ at the same transmission rate. In Fig. 4 (a), we also observe that PS with short blocklengths can also slightly increase the nonlinear tolerance, and thus, the SNR $\mathrm{Sff}_{\text {. The phenomena }}$ has been reported in 22 and very recently in 13 .

\section{Conclusions}

We have studied the performance of various signal shaping schemes in the presence of fibre nonlinearities. All formats have with the same spectral efficiency (7 bit/4D-sym), however, they differ greatly in the distribution of their symbol energies. 4D symbol energy considerations showed that constant-modulus constellations reduce the $\mathrm{NLI}$ and that probabilistic shaping exhibits large SNR variations across symbols with different energies. The newly introduced 4D-OS128 format was shown to be able to trade-off linear and nonlinear tolerance giving SNR improvements with respect to PS-16QAM. This is achieved by introducing less 4D symbol energy variations in the transmit sequences that effectively mitigates fiber nonlinearities.

Acknowledgements: The work of B. Chen is supported by the National Natural Science Foundation of China (NSFC) under Grant 61701155. C. Okonkwo is supported in part by the Dutch NWO Gravitation Program: Research centre for Integrated Nanophotonics (Grant Number 024.002.033). The work of A. Alvarado is supported by the Netherlands Organisation for Scientific Research (NWO) via the VIDI Grant ICONIC (project number 15685).

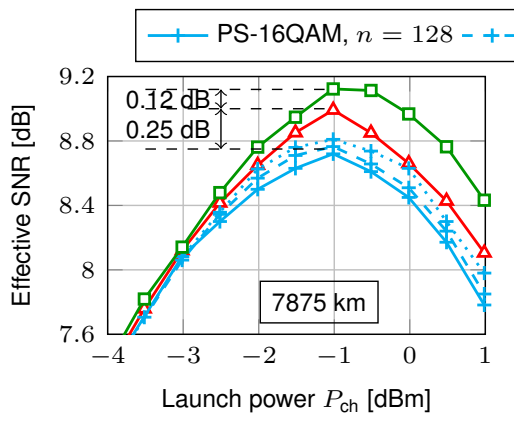

(a) Effective SNR vs. $P_{\text {ch }}$ at $7875 \mathrm{~km}$.

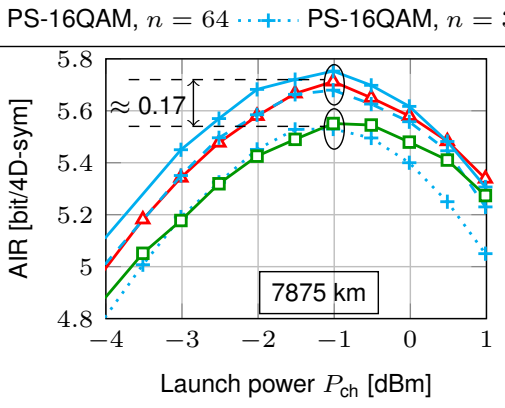

(b) AIR vs. $P_{\text {ch }}$ at $7875 \mathrm{~km}$.

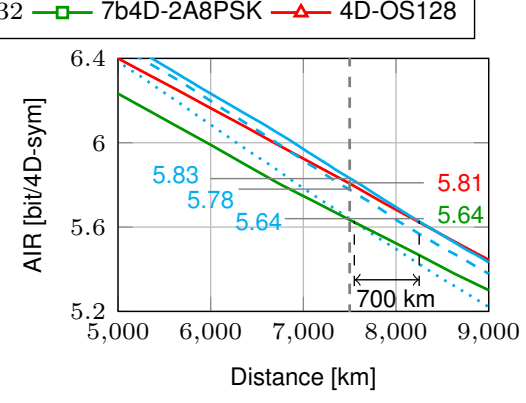

(c) AIR vs. distance at optimal $P_{\mathrm{ch}}$.

Fig. 4: Simulation results of multi-span optical fiber transmission with 11 WDM channels for three modulation formats with SE of 7 bit/4D-sym: 7b2D-4D2A8PSK, 4D-OS128 and PS-16QAM with three different blocklengths. 


\section{References}

[1] P. Poggiolini, G. Bosco, A. Carena, V. Curri, Y. Jiang, and F. Forghieri, "The GN-Model of Fiber Non-Linear Propagation and its Applications", J. Lightw. Technol., vol. 32, no. 4, pp. 694-721, Feb. 2014.

[2] P. Serena, A. Bononi, and N. Rossi, "The impact of the modulation dependent nonlinear interference missed by the Gaussian noise model", in 2014 Eur. Conf. on Opt. Commun. (ECOC), 2014.

[3] L. Galdino, G. Liga, G. Saavedra, D. Ives, R. Maher, A. Alvarado, S. Savory, R. Killey, and P. Bayvel, "Experimental Demonstration of Modulation-Dependent Nonlinear Interference in Optical Fibre Communication", in 2016 Eur. Conf. on Opt. Commun. (ECOC), 2016.

[4] A. Carena, G. Bosco, V. Curri, Y. Jiang, P. Poggiolini, and $F$. Forghieri, "EGN model of non-linear fiber propagation", Opt. Express, vol. 22, no. 13, pp. 1633516362, Jun. 2014

[5] R. Dar, M. Feder, A. Mecozzi, and M. Shtaif, "InterChannel Nonlinear Interference Noise in WDM Systems: Modeling and Mitigation", J. Lightw. Technol., vol. 33, no. 5, pp. 1044-1053, Mar. 2015.

[6] "Nokia PSE-V technical White Paper". [Online]. Available: https://onestore.nokia.com/asset/207220

[7] "1FINITY T600 Transport Blade". [Online]. Available: https : / / www . fujitsu . com/us / Images / 1FINITY T600-Data-Sheet.pdf

[8] J. Cho, S. Chandrasekhar, R. Dar, and P. J. Winzer, "Low-Complexity Shaping for Enhanced Nonlinearity Tolerance", in 2016 European Conference on Optical Communication (ECOC), 2016.

[9] T. Fehenberger, A. Alvarado, G. Böcherer, and N. Hanik, "On Probabilistic Shaping of Quadrature Amplitude Modulation for the Nonlinear Fiber Channel", J. Lightw. Technol., vol. 34, no. 21, pp. 5063-5073, Nov. 2016.

[10] F. Buchali, F. Steiner, G. Böcherer, L. Schmalen, P. Schulte, and W. Idler, "Rate Adaptation and Reach Increase by Probabilistically Shaped 64-QAM: An Experimental Demonstration", J. Lightw. Technol., vol. 34, no. 7, pp. 1599-1609, Apr. 2016.

[11] J. Renner, T. Fehenberger, M. P. Yankov, F. D. Ros, S. Forchhammer, G. Böcherer, and N. Hanik, "Experimental Comparison of Probabilistic Shaping Methods for Unrepeated Fiber Transmission", J. Lightw. Technol., vol. 35, no. 22, pp. 4871-4879, Nov. 2017.

[12] E. Sillekens, D. Semrau, G. Liga, N. A. Shevchenko, Z. Li, A. Alvarado, P. Bayvel, R. I. Killey, and D. Lavery, "A Simple Nonlinearity-Tailored Probabilistic Shaping Distribution for Square QAM", in 2018 Opt. Fiber Commun. Conf. (OFC), Mar. 2018.

[13] T. Fehenberger, D. S. Millar, T. Koike-Akino, K. Kojima, K. Parsons, and H. Griesser, "Analysis of Nonlinear Fiber Interactions for Finite-Length ConstantComposition Sequences", J. Lightw. Technol., vol. 38, no. 2, pp. 457-465, Jan. 2020.

[14] T. Fehenberger, H. Griesser, and J. Elbers, "Mitigating Fiber Nonlinearities by Short-Length Probabilistic Shaping", in 2020 Optical Fiber Communications Conference and Exhibition (OFC), 2020.
[15] K. Kojima, T. Yoshida, T. Koike-Akino, D. S. Millar, K. Parsons, M. Pajovic, and V. Arlunno, "NonlinearityTolerant Four-Dimensional 2A8PSK Family for 5-7 Bits/Symbol Spectral Efficiency", J. Lightw. Technol., vol. 35, no. 8, pp. 1383-1391, Apr. 2017.

[16] B. Chen, C. Okonkwo, H. Hafermann, and A. Alvarado, "Polarization-ring-switching for nonlinearitytolerant geometrically-shaped four-dimensional formats maximizing generalized mutual information", J. Lightw. Technol., vol. 37, no. 14, pp. 3579-3591, Jul. 2019.

[17] R. Dar et al., "On shaping gain in the nonlinear fiberoptic channel", in IEEE Int. Symp. on Inf. Theory, Jun. 2014, pp. 2794-2798.

[18] B. Chen, A. Alvarado, S. van der Heide, M. van den Hout, H. Hafermann, and C. Okonkwo, "Analysis and Experimental Demonstration of Orthant-Symmetric Four-dimensional 7 bit/4D-sym Modulation for Optical Fiber Communication", arXiv e-prints, 2020. [Online]. Available: https://arxiv.org/abs/2003.12712

[19] T. Fehenberger, D. S. Millar, T. Koike-Akino, K. Kojima, and K. Parsons, "Multiset-Partition Distribution Matching", IEEE Transactions on Communications, vol. 67, no. 3, pp. 1885-1893, Mar. 2019.

[20] G. Böcherer, P. Schulte, and F. Steiner, High Throughput Probabilistic Shaping with Product Distribution Matching, 2020. [Online]. Available: https : //arxiv . org/abs/1702.07510

[21] A. Ghazisaeidi, "A Theory of Nonlinear Interactions Between Signal and Amplified Spontaneous Emission Noise in Coherent Wavelength Division Multiplexed Systems", J. Lightw. Technol., vol. 35, no. 23, pp. 51505175, 2017.

[22] A. Amari, S. Goossens, Y. C. Gültekin, O. Vassilieva, I. Kim, T. Ikeuchi, C. Okonkwo, F. M. J. Willems, and A. Alvarado, "Introducing Enumerative Sphere Shaping for Optical Communication Systems with Short Blocklengths", J. Lightw. Technol., vol. 37, no. 23, pp. 59265936, Dec. 2019. 